\title{
To what extent is the new position statement of the American Diabetes Association (ADA) and the European Association for the Study of Diabetes (EASD) 'personalised'?
}

\author{
A. Ceriello • M. Gallo • S. Gentile • C. B. Giorda - A. De Micheli • \\ on behalf of Associazione Medici Diabetologi (AMD)
}

Received: 14 June 2012 / Accepted: 18 June 2012 /Published online: 17 July 2012

(C) Springer-Verlag 2012

Keywords Algorithm · Fasting glycaemia · Personalised · Postprandial hyperglycaemia $\cdot$ Self blood glucose monitoring

\author{
Abbreviations \\ AMD Associazione Medici Diabetologi \\ SMBG Self-monitoring of blood glucose
}

A. Ceriello $(\bowtie)$

Institut d'Investigacions Biomèdiques August Pi i Sunyer (IDIBAPS),

C/ Rosselló, 149-153,

08036 Barcelona, Spain

e-mail: aceriell@clinic.ub.es

\section{A. Ceriello}

Centro de Investigación Biomédica en Red de Diabetes y

Enfermedades Metabólicas Asociadas (CIBERDEM), Spain

URL: www.ciberdem.org/

\section{Gallo}

Oncological Endocrinology, University Hospital

San Giovanni Battista,

Turin, Italy

S. Gentile

Department of Geriatrics and Metabolic Disease,

Second University of Naples,

Naples, Italy

\section{B. Giorda}

Diabetes and Metabolism Unit,

ASL TO5,

Chieri, Turin, Italy

\section{A. De Micheli}

Ligurian Health Agency,

Genoa, Italy
To the Editor: The joint position statement of the American Diabetes Association (ADA) and the European Association for the Study of Diabetes (EASD) on the management of hyperglycaemia in type 2 diabetes [1] not only constitutes a revision of the previous algorithm [2], but also a paradigm shift in the concept of diabetes care: the aspect repeatedly emphasised in this document is 'patient-centred care'. The new ADA/EASD position statement is certainly innovative compared with its predecessor, because it includes many other therapeutic options based on patient-centred care with respect to sulfonylureas and basal insulin, when treatment with metformin alone has not achieved the therapeutic target [2]. The new algorithm takes into consideration the need for personalised treatment, and provides a series of recommendations about possible choices, highlighting the advantages and limitations of each therapeutic option. In this sense, the document appears to fail in its purpose, by focusing - as do all guidelines available to date - on a lengthy though highly detailed list of the possible therapeutic choices. Even though the authors define the new recommendations as being less prescriptive and not as 'algorithm-like' as its forerunners, it is still up to the physician to choose the most appropriate therapy for the patient. It seems likely that the authors thought of proposing different algorithms based on various aspects that vary over time (apart from those proposed to avoid weight gain, minimise cost and avoid hypoglycaemia), but realised that these would be highly complicated. An effective way of overcoming this problem would be to use an online interactive resource, as recently proposed with the algorithms relating to tailoring drug therapy for patients with type 2 diabetes issued by the Finnish Medical Society [3]. This computerised system allows people to quickly find subjects of interest according to their own particular clinical features, as well as to follow easy, step-by-step suggestions for additional therapeutic pathways. 
In the $2012 \mathrm{ADA} / \mathrm{EASD}$ position statement [1], moreover, the importance of controlling postprandial hyperglycaemia remains largely undervalued, despite being a key contributory factor to achievement of the $\mathrm{HbA}_{1 \mathrm{c}}$ goal. The International Diabetes Federation (IDF) has recently developed an updated version of its own guidelines for the management of postprandial glucose in diabetes [4], acknowledging the fundamental importance of targeting both fasting and postprandial plasma glucose as an important strategy for achieving optimal glucose control. It is very surprising that the ADA/EASD position statement neglects this aspect, particularly if we consider that the main defect in Asian patients is loss of the first phase of insulin secretion, rather than insulin resistance [5], which is why drugs like $\alpha$-glucosidase inhibitors are among the first therapeutic choices in these patients. This is even more surprising given that there are Asian populations in North America and Europe [6].

Finally, within the context of 'customising' therapy, it is questionable that initiation of insulin therapy must be carried out exclusively with basal insulin, not including the possibility of using insulin that deals with postprandial hyperglycaemia. Almost all of the studies comparing the two approaches have shown that rapid-acting insulin is better than basal insulin at achieving $\mathrm{HbA}_{1 \mathrm{c}}$ goals $<7 \%$ $(<53 \mathrm{mmol} / \mathrm{mol}$ ) [4], even if hypoglycaemia is more common. Is it possible that not one patient would benefit from receiving prandial insulin therapy instead of a long-acting insulin during an early phase of the disease? This seems unlikely given that, during the early stage of the disease, the loss of the first phase of insulin secretion, producing high levels of glycaemia after meals, is the key feature of the disease.

In the ADA/EASD position statement, self-monitoring of blood glucose (SMBG), is also completely overlooked unless insulin therapy starts. SMBG, the timing and frequency of which should be individualised to each person's treatment regimen and level of glycaemic control [7], allows people with diabetes and clinicians to obtain and use information about real-time plasma glucose levels. Furthermore, it facilitates timely intervention to achieve and maintain glycaemic control. Recent studies have shown that structured SMBG followed by therapeutic interventions result in greater $\mathrm{HbA}_{1 \mathrm{c}}$ reduction, greater weight reduction, and better lifestyle scores in people with non-insulin-requiring type 2 diabetes compared with treatment without structured SMBG [8].

Recently, the Italian Association of Diabetologists (Associazione Medici Diabetologi, AMD) has increasingly felt the need to develop personalised treatment plans for people with type 2 diabetes, which take the patient's individual profile into account and direct them, on the one hand, toward the objective of the safest possible glycometabolic control and, on the other, towards optimised therapeutic choices, i.e. those that represent the best compromise between efficacy, safety and therapeutic adherence. To achieve this goal, AMD has developed tailored therapeutic algorithms for some of the most common type 2 diabetes phenotypes. These take age, BMI, presence of macrovascular complications, hypoglycaemia risk and the co-existence of chronic renal failure into consideration [9]. Particular emphasis has been placed on exploiting information supplied via the rational use of home-based SMBG as a tool for optimising diabetes management, according to the prevalence of fasting/pre-prandial or postprandial hyperglycaemia within a structured decision-making process. In our opinion, this innovative approach, tailored to the real-world situation may facilitate more timely and appropriate treatment changes, counteracting clinical inertia.

These algorithms are available online as a browseroperated interactive version, both in English and Italian [10]. The reader can quickly locate the subject of interest according to his or her clinical features, and also easily follow a step-by-step suggested additive therapeutic pathway. Online publication facilitates timely updating of the recommendations, ensuring that all healthcare professionals have the latest version of the algorithms readily available at their office.

In our opinion, compared with the recent ADA/EASD position statement, the algorithm proposed by AMD offers suggestions that are more detailed and closer to the everyday decision-making processes carried out by every physician facing an individual patient with specific characteristics, and having to make therapeutic choices the most effective for that particular patient, or rather, for that individual person suffering from diabetes.

Duality of interest The authors declare that there is no duality of interest associated with this manuscript.

Contribution statement All the authors were equally responsible for the conception and design and drafting of the manuscript, and all approved the final version for publication.

\section{References}

1. Inzucchi SE, Bergenstal RM, Buse JB et al (2012) Management of hyperglycaemia in type 2 diabetes: a patient-centered approach. Position statement of the American Diabetes Association (ADA) and the European Association for the Study of Diabetes (EASD). Diabetologia 55:1577-1596

2. Nathan DM, Buse JB, Davidson MB et al (2009) Medical management of hyperglycaemia in type 2 diabetes mellitus: a consensus algorithm for the initiation and adjustment of therapy. A consensus statement from the American Diabetes Association 
and the European Association for the Study of Diabetes. Diabetologia 52:17-30

3. Virkamäki A, Saltevo J (2011) Finnish Current Care Guideline for Diabetes: interactive approach to improve individualised treatment. Diabetologia 54:1264-1265

4. International Diabetes Federation (2011) 2011 Guideline for management of postmeal glucose in diabetes. IDF, Brussels. Available from http://www.idf.org/2011-guideline-management-postmealglucose-diabetes, accessed 29 May 2012

5. Hsu WC, Boyko EJ, Fujimoto WY et al (2012) Pathophysiologic differences among Asians, native Hawaiians, and other pacific islanders and treatment implications. Diabetes Care 35:1189-1198

6. King GL, McNeely MJ, Thorpe LE et al (2012) Understanding and addressing unique needs of diabetes in Asian Americans, native Hawaiians, and pacific islanders. Diabetes Care 35:1181-1188
7. International Diabetes Federation (2009) Guideline on selfmonitoring of blood glucose in non-insulin treated type 2 diabetes. IDF, Brussels. Available from http://www.idf.org/guidelines/selfmonitoring, accessed 29 May 2012

8. Polonsky WH, Fisher L, Schikman CH et al (2011) Structured, self-monitoring blood glucose significantly reduces A1C levels in poorly controlled, noninsulin-treated type 2 diabetes: results from the Structured Testing Program study. Diabetes Care 34:262-267

9. Ceriello A, Gallo M, Armentano V, Perriello G, Gentile S, de Micheli A, on behalf of Associazione Medici Diabetologi (2012) Personalizing treatment in type 2 diabetes: a self-monitoring of blood glucose inclusive innovative approach. Diabetes Technol Ther 14:373-378

10. Italian Association of Medical Diabetologists-AMD (2011) Personalisation of therapy in type 2 diabetes. Available from http:// www.aemmedi.it/algoritmi/, accessed 29 May 2012 